Revue des patrimoines

\title{
Les « Entretiens du patrimoine » (1988-2001) ou la " grand-messe » des professionnels du patrimoine
}

The 'Entretiens du patrimoine' (1988-2001), a 'high mass' for heritage professionals

\section{Cécile Lestienne}

\section{OpenEdition}

\section{Journals}

Édition électronique

URL : http://journals.openedition.org/insitu/13582

DOI : 10.4000/insitu. 13582

ISSN : 1630-7305

\section{Éditeur}

Ministère de la culture

\section{Référence électronique}

Cécile Lestienne, «Les « Entretiens du patrimoine » (1988-2001) ou la « grand-messe » des professionnels du patrimoine », In Situ [En ligne], 30 | 2016, mis en ligne le 20 septembre 2016, consulté le 01 mai 2019. URL : http://journals.openedition.org/insitu/13582 ; DOI : 10.4000/ insitu.13582

Ce document a été généré automatiquement le 1 mai 2019.

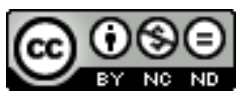

In Situ Revues des patrimoines est mis à disposition selon les termes de la licence Creative Commons Attribution - Pas d'Utilisation Commerciale - Pas de Modification 4.0 International. 


\section{Les « Entretiens du patrimoine » (1988-2001) ou la « grand-messe » des professionnels du patrimoine}

The 'Entretiens du patrimoine' (1988-2001), a 'high mass' for heritage professionals

\section{Cécile Lestienne}

1 Cette expression, " grand-messe des professionnels du patrimoine ", traduit l'impression que l'historien Pascal Ory ${ }^{1}$ ressentit lorsqu'il assista aux Entretiens de 1991. Le caractère professionnel semblait constituer l'essence même de ces rencontres alors pionnières pour le monde du patrimoine ${ }^{2}$.

2 C'est à l'occasion d'un grand «Forum du patrimoine », en 1987, qu'il fut décidé, sur une proposition du ministre de la Culture François Léotard, d'organiser des rencontres annuelles réservées à des spécialistes du patrimoine. Elles prirent le nom d'« Entretiens du patrimoine ${ }^{3}$ » en référence aux « Entretiens médicaux de Bichat », réunions organisées par la communauté médicale depuis 1947. Le principe général est le rassemblement, durant deux ou trois jours, de professionnels du patrimoine et, surtout à partir de 1994, d'universitaires et d'intellectuels attachés aux sciences humaines. Le contenu des conférences et des débats se retrouve dans des actes publiés par la direction du Patrimoine $e^{4}$.

3 Les premiers Entretiens (1988-1993) sont clairement établis pour servir d'outil de réflexion à un corps de professionnels du patrimoine assez hétérogène dans son origine, son organisation et sa formation. Ainsi cette manifestation participe-t-elle d'un mouvement plus général du ministère de la Culture en faveur de ces professionnels, avec la création, en 1990, d'un corps de conservateurs du patrimoine et de l'École nationale du patrimoine. À partir de 1994, une nouvelle formule est mise en place, avec une plus grande importance donnée à l'approche universitaire et intellectuelle dans les 
interventions. Le but manifeste, alors prôné par la direction du Patrimoine, est de «nourrir un corps de doctrine pertinent pour l'État ${ }^{5}$ ».

4 À une époque où la notion de patrimoine tend à s'élargir et à être perçue comme un " concept nomade ${ }^{6}$ ", ces colloques constituent bien un creuset où tentent de se fondre des réflexions, novatrices à l'aube du XXI ${ }^{\mathrm{e}}$ siècle, en matière de définition, de protection et de mise en valeur du patrimoine. Mais quels sont les objectifs poursuivis par ces rencontres ? Les professionnels du patrimoine en sont-ils les acteurs et les destinataires exclusifs? On essaiera d'abord de comprendre en quoi les Entretiens, entre 1988 et 1993, apparaissent comme un outil au service des professionnels du patrimoine, puis on examinera comment ces rassemblements deviennent, entre 1994 et 2001, un espace de théorisation, au service de l'État, de la notion de patrimoine ${ }^{7}$.

\section{Un outil de réflexion au service des professionnels du patrimoine (1988-1993)}

5 Entre 1988 et 1993, les contenus des Entretiens sont fortement axés sur des questions pratiques et techniques relatives à la conservation et à la mise en valeur du patrimoine en France et à l'étranger. Ces réflexions sont conduites, la plupart du temps, par des professionnels du patrimoine, à destination d'autres professionnels. C'est pourquoi l'emploi du terme « outil » apparaît approprié pour qualifier ces rencontres: leurs Actes peuvent être utilisés comme des « guides » dans les pratiques quotidiennes de ces acteurs. Jean-Pierre Bady, directeur du Patrimoine et instigateur de ces rencontres professionnelles, utilise à leur propos l'expression de « premier séminaire du patrimoine ${ }^{8}$ $"$ : il semble poser les Entretiens comme une première tentative, inédite, de réfléchir sur le patrimoine, même si d'autres rencontres sur ces sujets avaient eu lieu auparavant ${ }^{9}$.

\section{Le poids des « Monuments historiques »}

6 Une forte proportion de professionnels du patrimoine ${ }^{10}$, à hauteur de $75 \%$, se distingue parmi les intervenants. On y retrouve une certaine prépondérance des architectes en chef des monuments historiques et des inspecteurs des monuments historiques, qui ensemble ne représentent pas moins de $70 \%$ des professionnels intervenant aux Entretiens. En revanche, les conservateurs de musées sont très peu présents, un constat s'expliquant peut-être par le fait que ces professionnels relevaient de la direction des Musées de France et non de la direction du Patrimoine, organisatrice des Entretiens. La présence de professionnels des Monuments historiques révèle par ailleurs qu'un certain type de patrimoine, en l'occurrence le patrimoine dit "monumental», est au centre des discussions. En pleine période d'élargissement de la notion, on ne trouve pas, ou très peu, dans ces Entretiens d'avant 1994, d'acteurs dont la mission serait de conserver ou de mettre en valeur le patrimoine ethnologique ou industriel. Jean-Michel Leniaud ${ }^{11}$ fait observer, à juste titre, que l'expression "nouveaux patrimoines» ne résulte pas seulement d'une conscience nouvelle, mais de l'apparition de nouveaux médiateurs, associatifs ou élus, qui ne sont pas les habituels fonctionnaires spécialisés. Ces nouveaux médiateurs n'interviennent en aucun cas aux Entretiens. S'agissant du public, une évolution se dessine entre 1988 et $1993^{12}$. On dénombre à peu près 400 personnes aux Entretiens de 1991, alors qu'elles n'étaient que 120 en 1988. Les professionnels du patrimoine ne représentent pas loin de $55 \%$ de ces participants. Cela confirme l'idée 
selon laquelle les premiers Entretiens sont bien des rencontres dirigées par des professionnels du patrimoine, à destination d'autres professionnels. Architectes et conservateurs des monuments historiques constituent toujours l'écrasante majorité du public, loin devant d'autres professions, comme celle de conservateur à l'Inventaire général ou conservateur de musée, ou encore celle d'archéologue.

Ces premiers Entretiens permettent en outre à ces professionnels, qui n'ont peut-être pas assez l'habitude de travailler ensemble, de se rencontrer et de dialoguer. Ainsi Jean-Pierre Bady se réjouit-il de la "collaboration ${ }^{13}$ ", dans la préparation des interventions, entre architectes en chef et inspecteurs des monuments historiques. On a l'impression, en effet, que ces échanges n'existent pas alors dans le quotidien de ces acteurs. Jean-Michel Leniaud semble en tout cas le penser quand il compare ces différents corps de professionnels à « un conglomérat sympathique, entraîné depuis longtemps à ne pas collaborer ${ }^{14}$ ». De même, on perçoit, aux Entretiens, un réel désir de voir reconsidérés les professionnels de la restauration : le directeur du Patrimoine incite en 1989 les personnes présentes aux Entretiens à «faire un effort pour ne pas considérer [les restaurateurs] comme de simples auxiliaires, mais comme de véritables partenaires d'une politique du patrimoine ${ }^{15}$ ». Bien que les restaurateurs n'interviennent que très peu aux Entretiens, leur place est néanmoins importante dans l'assistance.

\section{Harmoniser les pratiques de conservation du « patrimoine monumental »}

8 Pour Jean-Pierre Bady, les premiers Entretiens représentent une «tribune" pour approfondir «les problèmes actuels concernant le patrimoine ${ }^{16}$ ». Et d'ajouter qu' "une politique du patrimoine ne se réduit pas à la demande et à l'obtention de crédits supplémentaires ${ }^{17}$ ». Le souci d'établir une " charte » de bonnes pratiques en matière de conservation se retrouve donc au cœur du débat. Cette réflexion technique s'inscrit alors dans le cadre de l'établissement de la loi-programme sur le patrimoine monumental, votée le 5 janvier 1988, qui avait pour objet de promouvoir la restauration et la mise en valeur des monuments historiques, mais aussi celles de grands sites archéologiques. Pour cela, un budget s'élevant à 5,1457 milliards de francs, réparti entre 1988 et 1992, fut approuvé. Durant les Entretiens, nombre d'exposés, comme celui sur l'amphithéâtre de Gand présenté par Michel Goutal ${ }^{18}$, portent sur des campagnes de restauration inscrites dans le cadre de cette loi-programme.

Dans ce contexte, on comprend mieux l'omniprésence, aux Entretiens, de sujets traitant d'objets patrimoniaux classés, ou inscrits, au titre des Monuments historiques. Par ailleurs, c'est surtout le patrimoine architectural, de l'Antiquité à l'époque moderne, qui est abordé : $66 \%$ des interventions lui sont consacrées. Les œuvres architecturales des XIX ${ }^{e}$ et $\mathrm{xx}^{\mathrm{e}}$ siècles n'y sont que peu ou pas abordées. À partir d'exemples concrets portant donc quasi exclusivement sur des monuments historiques, les intervenants aux Entretiens de 1988 à 1993 confrontent leurs points de vue sur leurs pratiques professionnelles. Ces exemples se fondent sur l'expérience même de l'exposant, car il est celui qui intervient généralement sur le terrain. Les enjeux de la réflexion s'orientent très souvent sur les différents partis de restauration qui peuvent être appliqués à l'égard d'un monument, partis qui font débat au sein de la communauté professionnelle depuis le XIX ${ }^{\mathrm{e}}$ siècle. Ainsi, note-t-on, aux Entretiens, des professionnels favorables à une intervention assez souple sur le monument, dans la lignée de John Ruskin ou de Camillo Boito, mais 
aussi des professionnels attirés par des reconstitutions peu documentées historiquement. Dans les Entretiens de 1988, Francis Salet propose, pour cette pratique de restauration ${ }^{19}$, la typologie suivante : la reconstitution peut être le fait de rapporter à un monument un décor qui en a été éloigné, mais aussi le fait de reconstituer un décor disparu, ou enfin, de reprendre un travail interrompu. Globalement, tous les procédés de reconstitution, voire de création, dans les monuments historiques, furent assez vivement rejetés par la profession. Ainsi, en 1990, Monique Chatenet ${ }^{20}$, conservateur à l'Inventaire, critique fortement les pratiques de restauration par restitution. Pour elle, « la restitution est [...] nécessairement aussi une invention ${ }^{21}$ ", et qui dit invention dit création à partir de l'œuvre originale.

10 Ce caractère pratique et professionnel des Entretiens tend à s'estomper à partir de 1994. Cette année entérine une rupture qui affecte l'ensemble des rencontres jusqu'en 2001.

\section{Un espace de théorisation de la notion de patrimoine (1994-2001)}

11 À partir de 1994, une rupture se fait sous l'impulsion de la nouvelle directrice du patrimoine, Maryvonne de Saint-Pulgent, nommée en juillet 1993. Cette dernière avait observé, dès son entrée en fonction, le " déficit doctrinal ${ }^{22}$ » de la direction, et l'incapacité de l'État à protéger efficacement son patrimoine ${ }^{23}$, un patrimoine perçu comme indéfinissable en raison de ses multiples formes et extensions. De là, peut-être, pour Maryvonne de Saint-Pulgent, cette volonté de revitaliser des Entretiens créés dans un autre contexte, afin d'en faire un véritable espace de théorisation de la notion de patrimoine, au service de l'État. Cette ambition s'inscrit dans le cadre du Rapport de la Commission d'étude de la politique de l'État, présidée par Jacques Rigaud, remis au ministre de la Culture le 18 octobre 1996. Ce Rapport préconisait alors que la politique culturelle, et en l'occurrence la politique patrimoniale, s'ouvrît bien plus à la recherche scientifique et à l'Université, afin de « corriger les effets stérilisants d'une extension non maîtrisée de la notion de patrimoine ${ }^{24} »$. Dans ce nouveau contexte, les Entretiens du patrimoine sont complètement repensés : la forme et les intervenants ne sont plus les mêmes, l'accent est mis sur une réflexion théorique, et le poids de l'État semble, dans le discours, beaucoup plus important. Ces nouveaux colloques dépassent par leur cadre et leur contenu le seul rôle d'outils pour les professionnels du patrimoine.

\section{Un « regard » sur le patrimoine partagé}

12 Ainsi, à partir de 1994, les professionnels du patrimoine partagent-ils l'affiche des Entretiens avec une autre catégorie de personnes : les universitaires et les « intellectuels ${ }^{25}$ ». Pour Maryvonne de Saint-Pulgent, il s'agit d'instaurer un «dialogue [...] entre les professionnels du patrimoine et le monde intellectuel ${ }^{26} »$. On peut d'abord observer ce "supplément d'âme" dans la présidence même des Entretiens: ce poste est en effet occupé majoritairement, entre 1994 et 2001, par des universitaires, et principalement par des historiens, comme Pierre Nora, directeur de la monumentale série d'ouvrages Les lieux de mémoire, en 1994, ou encore Jacques Le Goff, en 1997. D'une manière générale, les intellectuels représentent, entre 1994 et 2001, 39 \% de l'ensemble des intervenants aux Entretiens. Il s'agit d'une part très importante par rapport aux précédentes rencontres, où ils ne représentaient que $15 \%$ des interventions. Ces intellectuels sont la plupart du 
temps des universitaires, professeurs ou maîtres de conférence, mais aussi des directeurs de recherche dans des instituts tels que l'EHESS ou le CNRS. On remarque aussi la grande diversité des disciplines auxquelles se rattachent ces intellectuels qui relèvent, pour la plus grande part, des sciences humaines. Au sein de cette diversité, relevons néanmoins la prééminence de l'histoire et, dans une moindre mesure, de l'histoire de l'art. Cette nouvelle ambition n'est pas sans lien avec la création, en juin 1993, de l'association Momus . Se définissant comme un groupe de réflexion et d'action pour le patrimoine, tout en revendiquant vigoureusement son apolitisme, cette association «traduit cette sensibilité accrue de personnalités du monde intellectuel au patrimoine ${ }^{27}$ ». Parmi les membres fondateurs, on retrouve un certain nombre d'acteurs des Entretiens, comme Marc Fumaroli, Pierre Nora, ou Jean-Michel Leniaud.

13 Bien qu'un nouveau regard domine la réflexion, celui des professionnels du patrimoine n'est pas pour autant mis de côté. Entre 1994 et 2001, ces derniers représentent 33,2 \% de l'ensemble des personnes intervenant aux Entretiens. Le poids des conservateurs relevant de la spécialité « Monuments historiques » est important, comme celui des conservateurs de musée. Les architectes en chef des monuments historiques, quant à eux, ont vu leur présence baisser assez significativement. On retrouve enfin de nouveaux acteurs comme les archéologues, les archivistes ou les bibliothécaires. Ce ne sont donc plus les seuls professionnels relevant de la direction du Patrimoine qui sont conviés à intervenir aux Entretiens. Cette présence des professionnels reste surtout manifeste dans l'assistance. D'après plusieurs témoignages ${ }^{28}$, elle en constituerait le noyau principal. L'image d'une "grand-messe» annuelle du patrimoine durant laquelle se réunirait une sorte de corporation du patrimoine plus large qu'auparavant reste donc pertinente après 1994.

\section{Mettre en place une « doctrine » du patrimoine}

14 Le caractère théorique du contenu représente le deuxième ingrédient dans la nouvelle recette de ces rencontres. En effet, en conviant des intellectuels à intervenir sur l'histoire ou sur la définition de la notion de patrimoine, la direction du Patrimoine souhaitait que l'approche de cette dernière évolue. On débat donc sur des questions plus théoriques qu'aux Entretiens précédents. Les professionnels du patrimoine, quoique toujours présents, suivent aussi cette mutation, et tendent à présenter des sujets plus historiques et moins techniques au cours de leurs interventions. Ce besoin de réflexion théorique est né d'un constat : le patrimoine tend à devenir, à la fin du $\mathrm{xx}^{\mathrm{e}}$ siècle, une notion assez floue permettant de regrouper des objets fort différents dans leur statut patrimonial. Pour les intervenants comme pour l'assistance, il s'agit de comprendre les raisons de cette " explosion patrimoniale », et surtout de présenter des solutions, afin que ce phénomène ne dégénère pas. Une des questions centrales est de savoir si les institutions patrimoniales doivent tout protéger, au seul motif que ces objets, si disparates soient-ils, viennent du passé. La conclusion de Pierre Nora aux Entretiens de 1994 est à ce sujet édifiante : « Nous vivons le moment où l'explosion patrimoniale n'a pas fini de développer ses effets positifs alors que se font déjà sentir à plein ses retombées négatives, les effets de son implosion ${ }^{29}$.

15 Les intervenants proposent une approche historique de la notion de patrimoine, depuis le $\mathrm{XIX}^{\mathrm{e}}$ siècle jusqu'à nos jours, en s'interrogeant notamment sur les formes qu'elle a prises durant toute cette période. On essaye, par exemple, de situer le passage de l'emploi des termes de "monuments historiques", à celui de "patrimoine», ou encore de 
comprendre l'extension de la notion vers le «tout patrimoine » en la confrontant à deux autres concepts, la mémoire et l'identité. C'est dans cette optique que se tiennent les Entretiens de 1998, dont le thème est « L'abus monumental ", un thème derrière lequel de nombreux observateurs perçurent plutôt l'expression d'abus patrimonial ${ }^{30}$. Le but de Régis Debray, alors président du colloque, est de « lancer un appel civique à retrouver le vrai sens de monument ${ }^{31} »$, un sens qui, d'après lui, a disparu. Il dénonce une inflation des protections pour ce qu'il nomme le "monument-trace ", comme une fosse de mine ou la ligne Maginot, qui prendrait le pas sur le «monument-message », comme la Marseillaise ou le mur des Fédérés, ou le "monument-forme ", comme une cathédrale ou un musée ${ }^{32}$. L'interaction de la mémoire et de la recherche d'identité est, pour Pierre Nora, un des facteurs les plus importants de la constitution du patrimoine. Il demande toutefois à l'histoire d'en prendre le relais, et surtout de les contrôler. Et de conclure, en 1994, inversant les termes de la célèbre phrase de Rabelais, que " conscience sans sciences ${ }^{33}$ " ne serait que «ruine de l'âme ». En d'autres termes, s'il est légitime, pour l'auteur des Lieux de mémoire, de considérer certains objets comme "patrimoniaux ", la conscience de ce caractère patrimonial, fondé sur la mémoire et l'affirmation d'une identité, ne dispense pas de recourir à la science, en l'occurrence à l'histoire, pour en déterminer la valeur.

Mais à force de pointer les dérives de la notion de patrimoine, les Entretiens sont aussi fortement critiqués pour leur propre « dérive ${ }^{34}$ ». En effet, pour la revue Policultures, en 2001, on y parle « de moins en moins de patrimoine, et de plus en plus de la manière dont on en parle ${ }^{35}$ ». Par cette critique du caractère peut-être trop théorique des sujets présentés aux Entretiens, la revue juge problématique la trop grande importance réservée aux historiens dans les interventions. Elle réclame un retour au caractère technique des premiers Entretiens, caractère qui n'a pourtant pas disparu, mais a subi quelques transformations dans son approche. Ces sujets sont désormais envisagés de manière généraliste dans les interventions, intégrant, le plus souvent, le point de vue de l'historien d'art, ou développés lors des débats ou des tables rondes de ces nouveaux Entretiens.

17 Toute cette réflexion vise à créer une " doctrine ${ }^{36}$ » du patrimoine. Ce terme apparaît comme une clef pour comprendre ces Entretiens d'après 1994. Cela tient certainement à une volonté politique tendant à en faire, selon l'expression de Jean-Michel Leniaud, un « argumentaire [...] convaincant ${ }^{37}$ » pour la nouvelle politique entamée depuis 1993 par la direction du Patrimoine. Mais qu'en est-il de cette politique patrimoniale? Dans la revue Le Débat, en 1995, Maryvonne de Saint-Pulgent pointait que l'absence de définition claire de la notion de patrimoine entraînait une certaine carence d'une "politique de protection des monuments historiques ${ }^{38}$ ", une politique qui aurait dû être bien plus efficace. C'est dans ce cadre que se placent les interventions et les débats des nouveaux Entretiens. Ils doivent constituer les "fondements d'une politique nouvelle", pour que l'État ne «se repos[e plus] sur un modèle de trente ans ${ }^{39}$ ». Cet objectif est affirmé jusqu'en 2001 durant tous ces rassemblements.

\section{Une diffusion ambitieuse}

18 La nouvelle formule s'accompagne enfin d'une communication plus importante, qui affirme à plus grande échelle ce caractère de "grand-messe ». La presse est largement mobilisée ; la plupart des grands quotidiens nationaux publient un article à l'occasion de ces rencontres. On compte des articles dans des quotidiens nationaux comme Le Monde, La 
Tribune, Libération, et même dans un quotidien allemand, le Frankfurter Allgemeine, en 1997. À partir de 1998, d'autres médias furent utilisés, comme la radio France-Culture, une innovation révélant le souhait de toucher un public cultivé assez large, et plus seulement les professionnels du patrimoine. Grâce à cette communication poussée, les Entretiens du patrimoine se muent en un très grand rassemblement, avec plus de 1000 personnes dans le public à chaque édition, entre 1994 et 2001. Aux Entretiens de l'an 2000, le nombre de participants atteint le record de 1400 personnes. Parallèlement, la création d'une véritable collection éditoriale, les Actes des Entretiens du patrimoine, grave dans le marbre l'aspect inédit de ces assises françaises du patrimoine.

Ces rencontres constituent donc le reflet des préoccupations patrimoniales de la fin du siècle dernier. On se rend compte qu'elles ne furent pas limitées aux seuls professionnels du patrimoine, mais bien placées au cœur du débat public. Le patrimoine, notion «nomade » si contestée au début des années 1980, semble avoir intégré les habitudes de pensée au début des années 2000 , jusqu'à être envisagé à une échelle européenne. Les enjeux semblent d'ailleurs se déplacer à partir des Entretiens de 2003, où une « acceptation de la pluralité des réponses, fussent-elles contradictoires » est préférée à une proclamation de "doctrine unique ${ }^{40}$ ». Le but ne semble donc plus de donner une définition universelle du patrimoine, mais bien de confronter les avis et les appréciations quant au «légitime élargissement de la notion de monument historique ${ }^{41}$ ». Quelle que soit la formule mise en place, les Entretiens du patrimoine ont donc joué un rôle moteur dans cette nouvelle appréhension, appréhension qui nous semble si familière aujourd'hui.

\section{Chronologie des Entretiens du patrimoine} Coural. Debray.

1988: "L'ornementation architecturale en pierre dans les monuments historiques ", château de Fontainebleau, sous la présidence d'Yves Boiret.

1989 : « Architecture et décors peints », Amiens, sous la présidence de Jacques Thuillier.

1990 : « Faut-il restaurer les ruines? », Mémorial de Caen.

1991 : « De l'utilité du patrimoine », abbaye royale de Fontevraud.

1992 : « Meubles et immeubles », Abbaye aux Dames de Saintes, sous la présidence de Jean

1993 : «Le bois dans l'architecture », palais des congrès de Rouen, sous la présidence de Jean-Marie Pérouse de Montclos.

1994: "Sciences et consciences du patrimoine », Théâtre national de Chaillot, sous la présidence de Pierre Nora.

1995 : « Patrimoine, temps, espace : patrimoine en place, patrimoine déplacé », Théâtre national de Chaillot, sous la présidence de François Furet.

1997 : «Patrimoine et passions identitaires ", Théâtre national de Chaillot, sous la présidence de Jacques Le Goff.

1998 : «L'abus monumental ? ", Théâtre national de Chaillot, sous la présidence de Régis

2000 : «Villes d'hier, villes d'aujourd'hui en Europe », Théâtre national de Chaillot, sous la présidence de François Loyer. 
2001 : «Le regard de l'histoire; L'émergence et l'évolution de la notion de patrimoine au cours du $\mathrm{xx}^{\mathrm{e}}$ siècle en France ", Cirque d'hiver de Paris, sous la présidence d'Henry Rousso.

2003: «Du bon usage du patrimoine: utiliser pour conserver, ou conserver pour utiliser ? ", palais du Pharo à Marseille, sous la présidence de Bruno Foucart.

2005 : « Du jardin au paysage », Nancy, sous la présidence de Jean-Pierre Babelon.

2007: «Patrimoine de l'Europe, patrimoine européen?», Espace Pierre Cardin (Paris), sous la présidence de Jean Musitelli.

2011 : "Patrimoines et architectures des métropoles durables", théâtre de la Cité internationale (Paris), sous la présidence de Nicolas Michelin.

2013 : «Des monuments historiques aux patrimoines: le centenaire de la loi de 1913 », Bibliothèque nationale de France.

\section{NOTES}

1. - Entretien avec M. Pascal Ory, le 26/03/2006, au Centre d'histoire sociale du Xxe siècle.

2. - C'est dans le cadre de mes masters 1 et 2 d'histoire, soutenus à l'université Paris PanthéonSorbonne en 2006 et 2007, sous la direction de Pascale Goetschel et Pascal Ory, que j'ai étudié ces colloques organisés par le ministère de la Culture. Sujet: «Les Entretiens du patrimoine, 1988-2007, entre lieu de sociabilité et instrument politique ».

3. - Voir en fin d'article la chronologie des Entretiens du patrimoine de 1988 à 2013.

4. - Les Entretiens de 1988 à 1993 ont été publiés dans les Actes des colloques de la Direction du patrimoine, et ceux de 1994 à 2001, sous le titre d'Actes des Entretiens du patrimoine, aux éditions Fayard.

5. - Le regard de l'histoire: l'émergence et l'évolution de la notion de patrimoine au cours du $\mathrm{XX}^{e}$ siècle. Entretiens du patrimoine, Cirque d'hiver, Paris, 26-28 novembre 2001. Paris : Fayard/Éditions du patrimoine, coll. « Actes des Entretiens du patrimoine », 2003, p. 7.

6. - CHOAY, Françoise. L'allégorie du patrimoine. Paris : Éditions du Seuil, 1999 (1éd. 1992), p. 9.

7. - L'articulation placée au niveau des Entretiens de 1994 est nécessaire, car la forme et le discours de ces derniers, et de ceux qui suivent, changent radicalement des précédents. D'autre part, il ne nous a pas semblé pertinent de traiter les derniers Entretiens (2003, 2005, 2007, 2011 et 2013), étant donné qu'ils appartiennent à une histoire très récente et que leurs actes n'ont pas été publiés (excepté ceux de 2011).

8. - BOIRET, Yves (dir.). L'ornementation architecturale en pierre dans les monuments historiques. Entretiens du patrimoine, château de Fontainebleau, 1988. Paris : ministère de la Culture et de la Communication, coll. « Actes des colloques de la Direction du patrimoine », 1989, p. 5.

9. - Il y eut bien des réunions antérieures organisées par la direction du Patrimoine sur ces sujets, comme le colloque tenu à la Salpêtrière en 1984, s'intitulant "Les monuments historiques demain », ou le « Forum du patrimoine », organisé à la Villette, entre le 7 et le 11 octobre 1987.

10. - Dans le cadre de cet article, nous entendons par l'expression "professionnel du patrimoine" une personne, relevant ou non de l'administration publique, mais étudiant, conservant et valorisant le patrimoine, qu'il soit matériel ou immatériel. 
11. - LENIAUD, Jean-Michel. "Voyage au centre du patrimoine». Dans FABRE, Daniel et VOISENAT, Claudie (dir.). Domestiquer l'histoire : ethnologie des monuments historiques. Paris : éd. de la Maison des sciences de l'homme, 2000, p. 181-188.

12. - Statistiques réalisées à partir des listes des participants présents aux Entretiens du patrimoine entre 1988 et 1990.

13. - L'ornementation architecturale en pierre dans les monuments historiques. Op. cit.

14. - LENIAUD, Jean-Michel. Chroniques patrimoniales. Paris : Norma Édition, 2001, p. 465.

15. - Architecture et décors peints. Entretiens du patrimoine, Amiens, octobre 1989. Paris : direction du Patrimoine, coll. « Actes des colloques de la direction du patrimoine », 1990, p. 265.

16. - L'ornementation architecturale en pierre dans les monuments historiques. Op. cit., p. 270.

17. - Architecture et décors peints. Op.cit.

18. - Faut-il restaurer les ruines? Entretiens du patrimoine, Mémorial de Caen, novembre 1990. Paris : direction du Patrimoine, coll. « Actes des colloques de la direction du patrimoine », 1991.

19. - L'ornementation architecturale..., Op. cit, p. 140.

20. - CHATENET, Monique. «Les pièges de la restitution ». Dans Faut-il restaurer les ruines? Paris : direction du Patrimoine, 1990, p. 214-217.

21. - Ibid., p. 215.

22. - « Quelle politique du patrimoine?». Entretien avec Maryvonne de Saint-Pulgent. Le Débat, n o 84, mars-avril 1995, p. 171.

23. - POIRRIER, Philippe. «Politique affichée/politique discutée...». Dans VADELORGE, Loïc, POIRRIER, Philippe (dir.). Pour une histoire des politiques du patrimoine. Paris : Comité d'histoire du ministère de la Culture/La Documentation française, 2003.

24. - POIRRIER, Philippe (éd.). Les politiques culturelles en France. Paris: La Documentation française, 2002, p. 523.

25. - Pour la commodité de l'exposé, j'utiliserai le terme générique d'« intellectuels », pour désigner les intervenants, au colloque, issus des mondes universitaires et intellectuels, même si, bien entendu, des professionnels du patrimoine répondraient parfaitement à cette qualification. De plus, ceci n'est pas seulement une simplification de mon fait, mais l'expression que les directeurs du Patrimoine successifs utilisent pour les définir, dans chaque introduction aux Entretiens.

26. - Patrimoine, temps, espace. Entretiens du patrimoine, Théâtre national de Chaillot, Paris, 22-24 janvier 1996. Paris: Fayard/Éditions du patrimoine, coll. «Actes des Entretiens du patrimoine ", 1997, p. 8.

27. - POIRRIER, Philippe. « Politique affichée, politique discutée... ». Op. cit., p. 589.

28. - Dans l'émission d'Emmanuel Laurentin, le 10 janvier 1997, Jacques Le Goff précise que la majorité de la salle, aux Entretiens de 1997, était occupée par des " acteurs du patrimoine »; voir «Le patrimoine, une obsession française ». Emmanuel Laurentin (prod.), Émission « La fabrique de l'histoire », France-Culture, le 08/03/2004.

29. - Science et conscience du patrimoine. Entretiens du patrimoine, Théâtre national de Chaillot, Paris, 28-30 novembre 1994. Paris : Fayard/Éditions du patrimoine, coll. « Actes des Entretiens du patrimoine ", 1997, p. 391.

30. - Entretien avec M. Jean-Pierre Bady, qui avait participé aux Entretiens de 1998, le 22/03/2007.

31. - « Patrimoine : Le danger de l'inflation des protections ». Le Moniteur, 13/11/1998.

32. - DEBRAY, Régis. "Le monument ou la transmission comme tragédie ». Dans DEBRAY, Régis. L'abus monumental? (Entretiens du patrimoine, Théâtre national de Chaillot, Paris, 23-25 novembre 1998). Paris: Fayard/Éditions du patrimoine, Coll. «Actes des Entretiens du patrimoine ", 1999, p. 11-22.

33. - Sciences et conscience..., Op. cit., p. 399.

34. - « La lente dérive des Entretiens du patrimoine ». Policultures, décembre 2001. 
35. - Ibid.

36. - Le terme est utilisé à peu près dans toutes les introductions des Entretiens à partir de 1994 .

37. - LENIAUD, Jean-Michel. Les archipels du passé, le patrimoine et son histoire. Paris : Fayard, 2002, p. 310.

38. - Ibid.

39. - NORA, Pierre (dir.). Sciences et conscience du patrimoine. Entretiens du patrimoine, Théâtre national de Chaillot, Paris, 28-30 novembre 1994. Paris: Fayard/Éditions du patrimoine, coll. "Actes des Entretiens du patrimoine ", 1997, p. 10.

40. - Présentation des Entretiens du patrimoine 2003, par Bruno Foucart, dans: www.edp2003.culture.gouv.fr/culture/actualites/entretiens2003/communique htm. [Consulté le 20/05/2014].

41. - Ibid.

\section{RÉSUMÉS}

C'est en 1987 que le ministre de la Culture, François Léotard, annonça la création des Entretiens du patrimoine, rencontres annuelles dont le principe était le rassemblement de professionnels du patrimoine et, surtout à partir de 1994, d'universitaires et d'intellectuels attachés aux sciences humaines, pour débattre de questions relatives à la conservation ou à la définition du patrimoine. D'une durée moyenne de deux ou trois jours, ils se déroulèrent dans différentes villes de province de 1988 à 1993, puis à Paris de 1994 à 2001. Le contenu des conférences et des débats fut publié dans les Actes des colloques de la Direction du patrimoine, de 1988 à 1993, puis sous le titre d'Actes des Entretiens du patrimoine, aux éditions Fayard, à partir de 1994. Conçus, dès 1988, comme un outil au service de la formation des professionnels, les Entretiens n'en furent pas moins mués, à partir de 1994, en un espace de théorisation de la notion de patrimoine.

In 1987, the Minister of Culture, François Léotard, announced the creation of the 'Entretiens du patrimoine', annual 'conversations' bringing together heritage professionals and, in particular, since 1994, academics and intellectuals from the humanities, in order to discuss issues raised by the conservation and the identification of the heritage. Lasting two or three days on average, these meetings took place in different provincial towns between 1988 and 1993, then in Paris from 1994 to 2001. The proceedings of the lectures and debates were published from 1988 to 1993 as 'Actes des colloques de la Direction du patrimoine', then, from 1994 onwards, under the series title of 'Actes des Entretiens du patrimoine'. Initially designed as a 'tool' for professional training, these 'Entretiens' have evolved, since 1994, into a public forum were notions of heritage are debated and theorised.

\section{INDEX}

Mots-clés : patrimoine, Monuments historiques, conservation, conservateur du patrimoine, restauration, professionnel du patrimoine, intellectuel, universitaire, Direction du patrimoine Keywords : heritage, historic monuments, heritage curator, restoration, heritage professional, intellectual, academic, heritage directorate 


\section{AUTEUR}

\section{CÉCILE LESTIENNE}

Attachée de conservation du patrimoine, Responsable du pôle Inventaire du patrimoine, Archives départementales du Val-d'Oise cecile.lestienne@valdoise.fr 